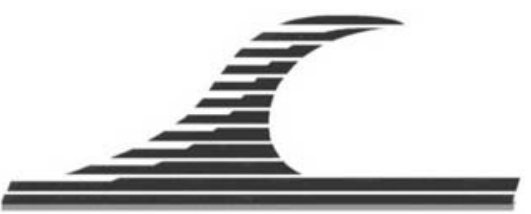

\title{
Historique du bloc artificiel utilisé dans les digues à talus
}

\author{
Iman SAFARI $^{1}$, François ROPERT ${ }^{2}$, \\ Dominique MOUAZÉ ${ }^{1}$, Alexander EZERSKY ${ }^{1}$
}

1. Université de Caen, UMR 6143 CNRS -M2C, 2-4 rue des Tilleuls, 140000 Caen, France.

safari.iman@gmail.com

2. Société Soframarp,

4, Sq Jean Mermoz 60200 Compiègne, France.

\section{Résumé :}

Parmi l'ensemble des différentes parties composant une digue à talus maritime, la carapace représente l'élément clé de la stabilité de la structure. Elle se compose soit d'enrochements naturels soit de blocs artificiels en béton. Le développement des blocs artificiels performants a fait l'objet de multiples travaux de la part de chercheurs du monde entier. Il s'agissait principalement de proposer des formes optimisées conduisant à une grande stabilité hydraulique autorisant par là de faibles consommations de béton et donc des économies substantielles. Le présent papier vise, après avoir en avoir fait une présentation globale, à proposer une nouvelle grille de classification des principaux blocs utilisés et à en dégager les intérêts respectifs tout en mentionnant les éventuelles incertitudes qui s'attachent à leur utilisation.

Soumis le 5 mars 2009, accepté le 10 décembre 2010, en ligne le 23 septembre 2013.

La seule version examinée est celle écrite en français. La ou les autres versions n'étant pas examinées par le comité de rédaction de la revue, sont donc publiées sous l'entière responsabilité du ou des auteurs.

Pour citer cet article :

SAFARI I., ROPERT F., MOUAZÉ D., EZERSKY A. (2013). Historique du bloc artificiel utilisé dans les digues à talus. Revue Paralia, Vol. 6, pp 4.1-4.14.

DOI:10.5150/revue-paralia.2013.004 (disponible en ligne - http://www.paralia.fr - available online) 


\section{Introduction}

Parmi l'ensemble des différentes parties composant une digue à talus maritime, la carapace représente un élément clé de la stabilité de la structure. Elle se compose soit d'enrochements naturels soit de blocs artificiels en béton lorsque l'intensité de la houle requiert des poids d'éléments naturels que les carrières disponibles ne sont pas en mesure de fournir. Les blocs artificiels, de part leur forme plus ou moins complexe, sont caractérisés par un coefficient de stabilité élevé.

La conception et le développement des blocs artificiels performants a fait l'objet de multiples travaux de la part de chercheurs du monde entier depuis 50 ans. Il s'agissait principalement de proposer des formes optimisées conduisant à une grande stabilité hydraulique autorisant par là de faibles consommations de béton et donc des économies substantielles. C'est la raison pour laquelle, les types de blocs artificiels se comptent aujourd'hui par dizaines, même si, bon nombre d'entre eux n'ont donné lieu qu'à de rares applications en nature.

La grande variété de ces blocs porte, non seulement sur les formes, mais aussi sur les modalités de pose qui jouent un rôle essentiel. La classification générale distingue parmi l'ensemble des enrochements artificiels, les blocs massifs, les blocs compacts et les blocs à formes élancées.

\section{Vue d'ensemble historique}

En comparaison des autres types d'ouvrages de défense contre la houle que sont les digues verticales et les digues mixtes, les digues à talus représentent la catégorie de structures la plus volontiers utilisée. Cette préférence tient sans doute à de multiples avantages tels qu'une relative facilité de mise en œuvre, leur caractère peu réfléchissant, la progressivité des dommages ainsi que leur possible réparation.

La figure 1 représente un profil-type schématique d'une digue à talus. La carapace constitue l'élément de protection principal de la structure vis-à-vis de l'agression des houles. Cette couche peut être composée de simples enrochements naturels lorsque l'intensité de ces dernières demeure assez limitée ou dans l'hypothèse où le site offre une disponibilité en calibre d'éléments naturels particulièrement forte. Dans tous les autres cas, il faut recourir aux blocs artificiels en béton.

On considère que le Tétrapode, conçu et breveté par Neyrpic en 1950, représente le premier bloc artificiel si l'on exclut les formes simples telles que le cube ou le parallélépipède.

C'est le bloc le plus utilisé dans le monde. Placé en deux couches d'une porosité voisine de $50 \%$, il présente des performances remarquables en matière de limitation de franchissement.

A la fin des années 1950, les États-Unis ont développé le Tribar qui ressemble à un trépied en béton. De nombreux autres types de blocs ont suivi comme le Cube modifié (US, 1959), l'Hexapode (US, 1959), le Stabit (U.K., 1962), l'Akmon et le Tripode 
(Pays-Bas, 1962), le Dolos (Afrique de Sud, 1963) et le Cube rainuré (Antifer, France, 1973). Dans un premier temps, les ingénieurs et chercheurs se sont en priorité concentrés sur la performance hydraulique des blocs. Il s'agissait de proposer des formes permettant d'atteindre une forte stabilité d'équilibre des éléments vis-à-vis des sollicitations hydrauliques générées par la houle, le plus souvent, au moyen d'une forte imbrication de blocs composant une couche présentant une porosité aussi grande que possible. Un bloc performant signifiait donc une consommation réduite de béton. De la même façon, les performances du bloc vis-à-vis du franchissement étaient également prises en considération puisqu'elles influençaient la hauteur de l'ouvrage. De fait, les blocs aux formes élancées bénéficiaient d'un grand intérêt de la part des praticiens. Toutefois, les dommages subis, en 1978 au Portugal, par la digue de Sines composée de Dolos allaient souligner l'importance d'un problème jusqu'alors trop largement ignoré. Il s'agit de la vulnérabilité structurelle des grands blocs aux formes élancées susceptibles de subir des ruptures. Ainsi, depuis cette époque, il semble que la tendance se soit orientée vers des formes plus compactes beaucoup plus aptes à résister aux chocs que les blocs sont amenés à subir.

Une innovation significative a été apportée en 1980 par SOGREAH en proposant l'Accropode, un bloc pouvant se positionner en une seule couche au lieu des deux épaisseurs habituelles pour les blocs concurrents.

De plus, la mise en place peut s'effectuer sur une pente plus forte. Les économies induites par ce nouveau produit expliquent le succès qu'il a connu.

D'autres blocs ont plus récemment vu le jour, qu'il s'agisse par exemple du Core-loc, du A-Jack ou du X-bloc.

Le Core-loc a ceci de particulier qu'un des soucis de ses concepteurs était de pouvoir l' utiliser en réparation de carapace composée de Dolos.

Enfin, les concepteurs d'un des blocs les plus récemment développés, le X-bloc, mettent en avant des avantages en matière de pose, le bloc étant censé s'imbriquer avec ses voisins de façon relativement aisée sans qu'il soit nécessaire d'apporter un soin extrême pour parvenir à cette fin. La figure 2 donne un bref aperçu de certaines formes de blocs utilisées dans le monde.

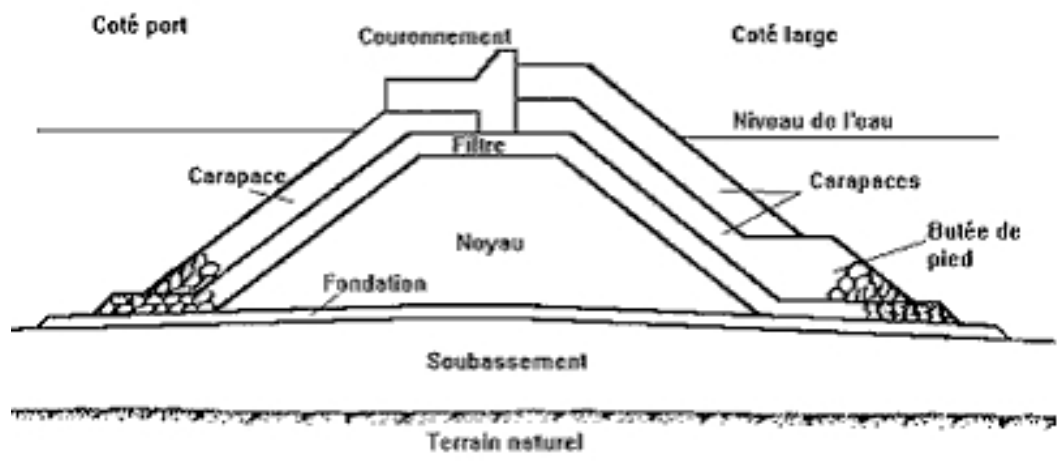

Figure 1. Coupe-type schématique d'une digue à talus. 


\section{Classification des blocs artificiels}

La grande variété relative à ces blocs porte, non seulement sur les formes, mais aussi sur les modalités de pose qui jouent un rôle essentiel. En ce qui concerne le paramètre "forme", on peut adopter la classification suivante (BURCHARTH \& HUGHES, 2002) :

1. Massive (Cube, Cubes rainurés,...)

2. Compacte (Accropode, X-bloc, Akmon, etc.)

3. Elancée (Tétrapode, Dolos, Core-loc, Hamabit, Stabit, etc.)

4. Perforée (Haro, Sea-bee, Shed, etc.)

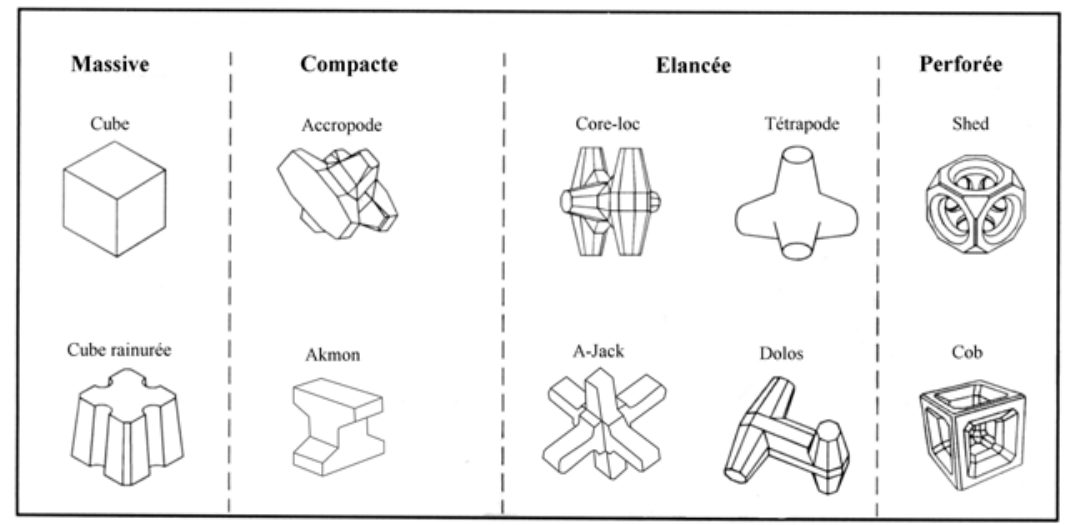

Figure 2. Différentes formes de blocs artificiels.

En ce qui concerne l'équilibre, les forces stabilisatrices des blocs qui s'opposent aux efforts hydrauliques générés par les vagues résultent, outre de leur frottement sur le support de pose, de leur poids et du contact qui s'établit entre les blocs voisins. Suivant le type d'élément, la nature de ce dernier contact peut être apparentée à un enchevêtrement c'est à dire une imbrication des blocs, comme dans le cas des Dolos, par exemple. Ce contact peut aussi se réduire à un simple frottement périphérique, comme dans le cas des cubes placés en une seule couche notamment.

Le classement proposé ici retient trois aspects principaux entrant dans les mécanismes favorables à la stabilité des blocs. Il s'agit de :

1. La masse

2. L'imbrication

3. Le frottement latéral

Naturellement, pour la majorité des blocs, les trois aspects interviennent. Toutefois, le classement s'est efforcé de souligner le mécanisme apparaissant comme dominant.

Les modalités de pose constituent un autre aspect important qu'il convient de considérer en matière d'utilisation de blocs artificiels. C'est particulièrement fondamental dans le cas des blocs dont les performances résultent en priorité de l'imbrication et du frottement latéral. 
La classification ici présentée distingue les trois types de placement suivants (voir tableau 1) :

1. Sans plan de pose

2. Avec plan de pose

3. Blocs rangés

Il convient d'expliciter cette distinction :

- Sans plan de pose signifie que les blocs sont simplement juxtaposés sans que la position de chaque bloc sur le support ne soit définie. En France, il n'y a quasiment que les enrochements naturels qui soient mis en place sans plan de pose. En ce qui concerne les blocs artificiels, les cubes placés en une seule couche appartiennent à la présente catégorie. Toutefois, même si la position des cubes n'est pas définie, en revanche, la densité de pose des blocs, c'est-à-dire le nombre d'éléments par unité de surface, constitue un paramètre de dimensionnement de ce type de carapace.

- Avec plan de pose signifie ici que la position du centre de gravité de chaque bloc composant la carapace est précisément définie. En revanche, l'orientation dans l'espace du bloc peut, et même dans certains cas, doit être aléatoire.

C'est la grande majorité des blocs artificiels, placé normalement en deux couches mais parfois sur une seule couche.

- Blocs rangés signifie ici que, non seulement la position du centre de gravité est définie, mais aussi que l'orientation des blocs est précisée. En fait, les blocs sont le plus souvent serrés les uns contre les autres et posés à plat sur le filtre support. Il faut préciser que ces blocs sont, dans leur grande majorité, perforés. S'ils ne l'étaient pas, il se produirait un différentiel de pression très préjudiciable à leur stabilité de part et d'autre de la carapace. Dans le cas de ces blocs, c'est le frottement latéral qui est le facteur stabilisant. Ce type de blocs est généralement placé sur une couche pour former un revêtement continu.

Comme on peut le constater, le choix offert par l'ensemble des produits disponibles est très riche. Toutefois, en pratique, les cultures locales ou nationales pèsent fortement sur les pratiques retenues. Ainsi, en France, il n’y a plus guère, depuis les années 90, que deux types de blocs qui soient utilisés à grande échelle pour les projets d'importance, l'Accropode et le Cube rainuré.

Naturellement, des cubes simples ou des parallélépipèdes peuvent encore être produits dans la mesure des besoins en entretien d'ouvrages existants généralement anciens. Même les tétrapodes qui ont connu un grand intérêt jusqu'au début des années 80 ne sont pratiquement plus utilisés en France.

Afin de tenter de faire une synthèse comparative de la majorité des blocs utilisés, nous avons dressé ci-dessous un tableau de valeurs des principaux types d'éléments. Il s'agit d'une évaluation très sommaire donnant des tendances de groupe à l'intérieur desquels les blocs peuvent néanmoins présenter des performances très différentes. Nous avons choisi de retenir les critères suivants (tableau 2) : 
1. la stabilité hydraulique

2. la performance en termes de franchissement

3. la résistance structurelle

4. la facilité de mise en œuvre

5. la souplesse, il s'agit de la capacité de la carapace à épouser les déformations du corps de l'ouvrage, tels que les tassements généraux ou différentiels ainsi que les irrégularités géométriques du support de carapace.

6. la sécurité

7. l'économie

Tableau 1. Classification de blocs artificiels par critères de pose, forme et stabilité.

\begin{tabular}{|c|c|c|c|c|c|}
\hline \multirow[b]{2}{*}{ Disposition } & \multirow[b]{2}{*}{ Forme } & \multirow{2}{*}{$\begin{array}{c}\text { Nombre } \\
\text { de } \\
\text { couches }\end{array}$} & \multicolumn{3}{|c|}{ Facteur de stabilité } \\
\hline & & & La masse & L'enchevêtrement & $\begin{array}{l}\text { Le frottement } \\
\text { périphérique }\end{array}$ \\
\hline \multirow{2}{*}{$\begin{array}{c}\text { Sans plan } \\
\text { de pose }\end{array}$} & \multirow{2}{*}{ Massive } & Une & Cube, & & \\
\hline & & Deux & Tetrahedron & & \\
\hline \multirow{6}{*}{$\begin{array}{c}\text { Avec plan } \\
\text { de pose }\end{array}$} & \multirow{2}{*}{ Massive } & Une & & & \multirow{2}{*}{ cube rainuré } \\
\hline & & Deux & & & \\
\hline & \multirow{2}{*}{ Elancée } & Une & & Core-loc & \\
\hline & & Deux & & $\begin{array}{c}\text { Tétrapode, Tribar, } \\
\text { Stabit, Dolos, } \\
\text { Quadripode, Hexapode }\end{array}$ & \\
\hline & \multirow{2}{*}{ Compacte } & Une & & $\begin{array}{c}\text { Accropode, Xbloc, } \\
\text { Gassho }\end{array}$ & \\
\hline & & Deux & Akmon, Toskane & $\begin{array}{c}\text { Tripod, Akmon, } \\
\text { Toskane }\end{array}$ & \\
\hline \multirow{3}{*}{$\begin{array}{l}\text { Blocs } \\
\text { rangés }\end{array}$} & Elancée & Une & Tribar & $\begin{array}{c}\text { Tribar, A-Jack, } \\
\text { Hamabit }\end{array}$ & \\
\hline & \multirow{2}{*}{ Compacte } & Une & & & $\begin{array}{c}\text { Cob, Seabee, Shed, } \\
\text { Diahitis, } \\
\text { Hollow cube, Diode }\end{array}$ \\
\hline & & Deux & $\begin{array}{c}\text { Cube perforé, } \\
\text { Haro }\end{array}$ & & \\
\hline
\end{tabular}

Tableau 2. Synthèse comparative des principaux types de blocs (sources multiples).

\begin{tabular}{lccccccc}
\hline Blocs & $\begin{array}{c}\text { Hydrau- } \\
\text { lique }\end{array}$ & $\begin{array}{c}\text { Franchi- } \\
\text { ssement }\end{array}$ & Structure & $\begin{array}{c}\text { Mise en } \\
\text { oeuvre }\end{array}$ & Souplesse & Sécurité & Economie \\
\hline Massive & - & + & ++ & + & + & ++ & -- \\
Elancé (2 ouches) & + & + & - & - & + & -+ & - \\
Compact (1 couche) & + & - & + & -+ & - & -+ & + \\
Rangé (1 couche) & ++ & -- & - & -- & -- & -+ & -+ \\
\hline
\end{tabular}


Cette appréciation appelle quelques commentaires :

- Nous avons considéré que les blocs rangés, notamment lorsqu'ils sont perforés présentent les meilleures performances hydrauliques. En revanche, comme cette performance s'obtient grâce au contact étroit obtenu entre les éléments voisins, il importe que la mise en œuvre initiale permette effectivement ce contact et qu'au cours de la vie de l'ouvrage, ce contact ne vienne pas à se relâcher.

- Le signe -+ signifie que nous ne nous sommes pas déterminés soit en raison de la trop grande variation du critère à l'intérieur du groupe (mise en œuvre), soit en raison de l'insuffisance des données à notre disposition (économie), soit en raison de la forte dépendance à d'autres critères (sécurité).

\section{Présentation des formules de stabilité}

Les formules de stabilité représentent des relations entre les paramètres de la houle et les caractéristiques minimales des blocs susceptibles de maintenir leur équilibre vis-àvis de son action. Les forces auxquelles sont soumis les blocs composant une carapace résultent de phénomènes hydrauliques complexes difficiles à modéliser ou à schématiser ou même à mesurer. Il s'agit d'écoulements externes et internes à l'ouvrage particulièrement variables dans le temps et dans l'espace et dont les grandeurs sont influencées par une multitude de paramètres qu'une seule formule analytique peut difficilement prendre totalement en considération. Les champs d'écoulement dans la carapace notamment au voisinage du niveau de repos et plus particulièrement en cas de déferlement plongeant restent difficiles à appréhender tout comme leur évolution au cours d'une période. Ces obstacles théoriques pénalisent la fiabilité des évaluations des efforts hydrauliques s'exerçant sur les éléments. De même, la représentation des contacts entre les divers éléments de carapace relève d'un exercice délicat dans la mesure où le nombre, la nature et l'intensité des contacts d'un bloc isolé qui dépendent des arrangements ou dispositions particulières d'assemblage des éléments voisins, varient de façon aléatoire dans de larges proportions.

En dépit de ces difficultés théoriques, de multiples auteurs ont pu proposer des formulations assez simples dont certaines fournissent des résultats relativement fiables à condition toutefois, qu'elles soient effectivement utilisées dans leur domaine de validité. Certaines formules peuvent relever d'une analyse théorique s'appuyant sur des schématisations simplifiées de fonctionnement du système eau-structure. D'autres se présentent davantage comme une exploitation de résultats expérimentaux obtenus sur modèle.

D'un point de vue général, les formulations se sont, au cours des décennies, progressivement enrichies en intégrant l'influence d'un nombre croissant de paramètres. Au minimum, les formules prennent en compte la hauteur de la houle, les poids ou les masses volumiques des matériaux, les caractéristiques dimensionnelles des blocs, la forme des blocs et généralement l'angle que fait le talus de pose avec l'horizontale. 
Que les éléments représentent des enrochements naturels ou des blocs artificiels n'affecte en rien les approches intellectuelles suivies par les producteurs des formules. C'est pourquoi les expressions présentent généralement des formats sensiblement voisins ou strictement identiques.

Nous avons ici jugé utile de nous arrêter plus particulièrement sur la formule d'Iribarren pour son intérêt historique et sur celle de Hudson encore largement usitée de nos jours.

\subsection{La formule d'Iribarren}

Iribarren (IRIBARREN CAVANILLES, 1938) fut l'un des tous premiers à proposer une formule de stabilité. Sa formulation résulte d'une approche théorique. Il a considéré qu'une houle de hauteur donnée générait une vitesse proportionnelle à la racine carrée de cette hauteur. Estimant ensuite que l'effort hydrodynamique était proportionnel au carré de cette vitesse, il conclut donc à une proportionnalité entre la hauteur de la houle et la force déstabilisatrice de l'enrochement. Equilibrant cette force avec la projection sur le talus du poids déjaugé du bloc et le frottement de ce dernier sur son assise, il a proposé l'expression suivante :

$$
W=\frac{K \rho_{s} g H^{3}}{(\mu \cos \alpha \pm \sin \alpha)^{3}\left(\rho_{s} / \rho_{w}-1\right)^{3}}
$$

où $W$ est le poids du bloc, $H$ la hauteur de la houle incidente au pied de la digue, $\mu$ un coefficient de frottement, $\rho_{s}$ la masse volumique du bloc, $\alpha$ l'angle du talus, $K$ un coefficient sans dimension dépendant de la forme du bloc, $\rho_{w}$ la masse volumique de l'eau et $g$ l'accélération de la pesanteur.

A la montée de la vague, le poids et le frottement agissent de concert et c'est un signe plus $(+)$ qu'il faut considérer. A la descente de la vague, ces deux forces s'exercent en sens opposé, c'est le signe moins (-) qu'il faut retenir.

Utiliser la formule d'Iribarren revient, en somme, à réaliser un calcul statique de limite d'équilibre au glissement d'un bloc frottant sur son assise sous des efforts de traînée induits par des vitesses sensiblement parallèles au talus que génèrent la houle. Bien qu'il s'agisse d'une formule théorique, les coefficients $K$ et $\mu$ devaient être obtenus à partir de mesures expérimentales. En 1965, Iribarren a fourni des jeux de coefficients $K$ et $\mu$ à utiliser pour des enrochements, des cubes et des tétrapodes, avec des valeurs de $K$ très différentes en fonction du sens de mouvement de la vague sur le talus.

La formule peut également être écrite sous le format suivant :

$$
\frac{H}{\Delta D_{n}}=K^{\prime}(\mu \cos \alpha \pm \sin \alpha)
$$

où $\Delta$ est la densité déjaugée du bloc soit $\rho_{s} / \rho_{w}-1$, et $D_{n}$ son diamètre nominal donné par la racine cubique de son volume. 


\subsection{La formule d'Hudson}

Hudson (HUDSON, 1958 ; HUDSON, 1959) a réalisé toute une batterie d'essais en modèle réduit en houle régulière non déferlante sur des enrochements naturels et des tétrapodes. Dans sa recherche de détermination expérimentale des coefficients à utiliser dans la formule d'Iribarren (à la descente de la vague), il a constaté, outre la dispersion du coefficient de frottement, une grande variabilité du coefficient $K$ en fonction de la pente. Après exploitation de ses résultats expérimentaux, il a modifié dans l'équation d'Iribarren le terme représentant l'influence de l'angle du talus, pour finalement proposer la formulation alternative suivante :

$$
W=\frac{\rho_{s} g H^{3}}{\Delta^{3} K_{D} \cot \alpha}
$$

où $K_{D}$ est un coefficient sans dimension caractérisant la performance hydraulique du bloc. Il dépend notamment de la forme du bloc et du taux de dommage subi par la carapace. Cette formule serait, d'après son auteur, applicable pour des ouvrages de pente allant de 5/4 à 4/1. La modification apportée par Hudson conduit à transformer une équation théorique en une formule empirique.

En utilisant $\mathrm{D}_{\mathrm{n}}$ le diamètre nominal des blocs et $\mathrm{N}_{\mathrm{S}}$ le nombre de stabilité, la formule de Hudson peut être réécrite sous la forme :

$$
\frac{H}{\Delta D_{n}}=\sqrt[3]{K_{D} \cot \alpha}=N_{s}
$$

La formule d'Hudson reste encore aujourd'hui l'expression la plus utilisée en raison de sa facilité d'utilisation et de la richesse de l'accumulation des données relatives aux valeurs de $K_{D}$ portant sur de multiples blocs et dans de nombreuses configurations différentes.

\subsection{Les formules nordiques}

Les ingénieurs du nord de l'Europe ont développé, depuis la fin des années 80, toute une série de formules de stabilité de blocs sous le format général suivant :

$$
\frac{H_{s}}{\Delta D_{n}}=f\left(N, s_{0 m}, N_{0 d}, \phi\right)
$$

Il s'agit de formulations purement empiriques, s'appliquant spécifiquement à des blocs de nature donnée placés suivant une pente déterminée, celle associée le plus souvent au type de blocs concerné. La formule donne l'influence de la durée de la tempête via N, le nombre de vagues, de la période par l'intermédiaire de $\mathrm{s}_{\text {om }}$ la cambrure, du nombre de dommage $\mathrm{N}_{\text {od }}$ et éventuellement de $\phi$ la densité de pose. Les coefficients et exposants intervenant dans les formules résultent de calages réalisés à partir de résultats obtenus sur modèles réduits physiques.

A titre d'exemples, nous redonnons ci-dessous les formules que VAN DER MEER (1998b) à développé pour les cubes et les tétrapodes : 
Cubes : $\quad \frac{H_{s}}{\Delta D_{n}}=\left(6,7 \frac{N_{o d}^{0,4}}{N^{0,3}}+1,0\right) s_{o m}^{-0,1}$

Tétrapodes : $\frac{H_{s}}{\Delta D_{n}}=\left(3,75\left(\frac{N_{o d}}{\sqrt{N}}\right)^{0,5}+0,85\right) s_{o m}^{-0,2}$

\subsection{Autres formulations}

Nous venons de voir que les formules de stabilité sont très largement empiriques. Toutefois, des tentatives de modélisation plus théoriques sont régulièrement menées par divers chercheurs. Les efforts hydrodynamiques sont alors généralement estimés à l'aide de la formule de Morrison :

$F=\frac{1}{2} \rho_{w} A C_{D} U^{2}+\rho_{w} V C_{m} \frac{d U}{d t}$

Où $\mathrm{A}$ est la section du maître-couple du bloc, $\mathrm{V}$ son volume, $\mathrm{C}_{\mathrm{D}}$ et $\mathrm{C}_{\mathrm{m}}$ les coefficients de traînée et de masse ajoutée, $\mathrm{U}$ étant la vitesse du fluide et $\mathrm{dU} / \mathrm{dt}$ son accélération. Cette approche nécessite une connaissance précise des champs de vitesse et de leur évolution au cours du temps. Les auteurs y ont accès soit directement par la mesure sur modèle réduit (MELBY \& KOBAYASHI, 1996) soit par l'intermédiaire de modèles numériques (HATTORI et al., 1999).

Dans les deux cas, la détermination des coefficients de traînée et de masse ajoutée constitue une difficulté majeure, notamment à cause de leur variation en fonction du nombre de Reynolds. Si dans le cas des enrochements naturels, des mesures d'efforts en modèle réduit (TORUM, 1994) ont pu contribuer à mieux cerner les valeurs à appliquer, en revanche, les grandeurs relatives aux blocs artificiels demeurent très incertaines.

Les travaux menés par HATTORI et al. (1999) qui ont développé un modèle de stabilité de blocs Hamabit illustre cette difficulté. Les chercheurs ont utilisé un simple modèle monodimensionnel pour la simulation des écoulements à extérieur et à l'intérieur de la structure afin de déterminer, pour chaque pas de temps, les efforts hydrodynamiques exercés les blocs. Considérant un mouvement en rotation autour des points de contact entre les blocs, les auteurs ont pu déterminer les moments et zones critiques de rupture d'équilibre en précisant le nombre de stabilité en cohérence avec leurs résultats expérimentaux obtenus en canal à houle.

Ce type de calcul qui a été réalisé avec succès grâce à l'utilisation de paramètres judicieusement choisis porte sur un bloc monocouche en pose rangé, c'est-à-dire avec une géométrie de contacts définis. On imagine aisément la dispersion des résultats qui résulterait de calculs faits sur une pose aléatoire.

Dans le même esprit, VAN GENT (1996), en recourant à un modèle numérique bidimensionnel d'écoulement, a pu estimer la stabilité des enrochements composant 
l'enveloppe d'une digue à berme et reconstituer la forme d'équilibre de l'ouvrage sous l'influence d'une houle.

En dépit des incertitudes liées à sa mise en œuvre, le type de démarche mentionnée cidessus représente une voie prometteuse vers une meilleure compréhension du comportement spécifique des divers blocs. Toutefois, cette approche théorique semble devoir rester confinée au domaine de la recherche encore quelque temps avant que des outils numériques opérationnels puissent réellement évaluer, avec la fiabilité requise, la stabilité des blocs artificiels.

\subsection{Recoupement des formulations}

En reprenant le modèle physique de frottement adoptée par Iribarren, il est possible de démontrer la formule d'Hudson. On utilise la formule de Morrison en négligeant le terme d'inertie, considérant que l'instant critique d'instabilité correspond au maximum de la vitesse. Cette dernière grandeur est estimée, non plus proportionnelle à la racine carrée de la hauteur de la houle, mais à celle d'un run-up évalué suivant le format suivant $(\mathrm{LNH})$ :

$\frac{R_{U}}{H}=A\left(\frac{\tan \alpha}{\sqrt{s_{0}}}\right)^{0,5}$

où $\mathrm{s}_{0}=\mathrm{H} / \mathrm{L}_{0}=2 \pi \mathrm{H} /\left(\mathrm{gT}^{2}\right)$ représente une cambrure fictive de la houle

En considérant la phase critique de la montée de la vague sur le talus, les développements conduisent à la formule de stabilité suivante :

$\frac{H}{\Delta D_{n}}=C \sqrt[4]{s_{0}} \frac{(\cos \alpha+\sin \alpha)}{\sqrt{\tan \alpha}}$

En étudiant, sur la plage de variation retenue par Hudson, le terme fonction de $\alpha$, on s'aperçoit qu'il reste très sensiblement proche (moins de $3 \%$ ) de une fois et demi la racine cubique de $\cot \alpha$, ce qui conduit à :

$$
\frac{H}{\Delta D_{n}}=1,5 C \sqrt[4]{s_{0}} \sqrt[3]{\cot \alpha}
$$

On reconnaît l'expression d'Hudson :

$$
\frac{H}{\Delta D_{n}}=\sqrt[3]{K_{D} \cot \alpha}
$$

Avec $K_{D}=3,4 C^{3}\left(s_{0}\right)^{3 / 4}$

Il faut, d'autre part, faire observer que, dans la formule de VAN DER MEER (1988a) relative à la stabilité des enrochements naturels en déferlement plongeant, il apparaît un terme correspondant à l'inverse de la racine carrée du paramètre d'Iribarren. Or ce paramètre est lui-même inversement proportionnel à la racine carrée de la cambrure. On retrouve ainsi l'exposant égal à un quart de la cambrure figurant dans la formule de Van der Meer. Les travaux de Iribarren, de Hudson et de Van der Meer peuvent donc se recouper de cette façon. 


\section{Incertitudes}

Les projets réalisés au cours des dernières décennies ont montré clairement l'avantage économique indiscutable que représente la technique des blocs utilisés en monocouche. Toutefois des praticiens ont pu émettre, au sujet de sa mise en œuvre, diverses réserves ou mises en garde qui ne sont pas totalement infondées.

Tout d'abord, on peut considérer qu'en règle générale, plus un bloc est performant d'un point de vue hydraulique, plus la sécurité qu'il offre se réduit, si on considère, comme critère de sécurité, le rapport entre la hauteur de la houle conduisant à la ruine de l'ouvrage et celle correspondant au début de dommage. De même, les mouvements de sortie de blocs d'une carapace monocouche conduisent à une exposition directe du filtre. Pour pallier cette difficulté, il est nécessaire d'adopter des critères de dommages directement fonction du type de bloc retenu. De même, la fréquence des événements à considérer pourra également être modulée en fonction du bloc utilisé.

Ensuite, se pose un problème délicat à traiter, celui de la dispersion des résultats que l'on enregistre lorsqu'on réalise des essais sur modèle réduit. Cette dispersion est due principalement au fait qu'une structure composée d'un assemblage d'éléments séparés ne peut être reconstruite à l'identique. Les variations toucheront la perméabilité du noyau, l'agencement des filtres et surtout le placement des blocs de carapace.

Or, si sur modèle réduit, on enregistre déjà des écarts significatifs de résultats, que dire des différences auxquelles on peut s'attendre en nature ? En effet, sur un modèle réduit, il est assez aisé de respecter les règles et modalités de pose des blocs.

En nature, surtout sous la surface du plan d'eau éventuellement lorsque la hauteur de la houle n'est pas négligeable, le respect des règles de placement n'est pas systématique. Bien sûr, les promoteurs des blocs recommandent, pour le dimensionnement des ouvrages, des valeurs de $K_{D}$ beaucoup plus faibles que celles mesurées en modèle.

Mais, pour les projets d'importance, ce seront toujours les résultats enregistrés sur modèle réduit lors des essais spécifiques qui serviront de comportement de référence. Comment dans ces conditions, les aléas de pose lors du chantier peuvent-ils être pris en compte ? Dans certains cas, ces aléas sont intégrés à travers une majoration de $20 \%$ de $H_{S}$. Là encore, on peut considérer que ce type de difficulté se pose surtout pour les blocs performants dont l'imbrication ne s'obtient que par une attention toute particulière apportée à la pose.

Par ailleurs, l'introduction des blocs posés en une seule couche amène à s'interroger sur les règles de filtre à respecter entre les matériaux du noyau et ceux du filtre. En effet, l'interface entre le noyau et le filtre sera le siège d'écoulements internes plus intenses dans le cas d'une carapace monocouche. Si, de plus, la pose laisse apparaître des espaces localisés de filtre non protégés, le risque de perte des matériaux de taille réduite du noyau va s'en trouver accru. N'y a-t-il pas lieu, dans ces conditions, de proposer, en ce qui concerne le noyau, des règles de filtre plus sévères en monocouche que celles 
adoptées pour un ouvrage muni d'une carapace traditionnelle composée des deux épaisseurs de blocs?

Une autre incertitude concerne l'influence de l'usure progressive des blocs sur leur comportement. On sait que les enrochements naturels anguleux présentent une meilleure stabilité que ceux aux formes arrondies. Or, les blocs en béton voient assez souvent, au cours de leur vie, leurs arêtes progressivement s'estomper et leur forme générale s'arrondir. Par analogie avec le comportement des enrochements naturels, on peut s'interroger sur l'influence de cette modification morphologique sur la stabilité des blocs.

Enfin, nous avons mentionné le fait que les ingénieurs se préoccupaient désormais de la résistance interne du matériau. A de rares exceptions près, les professionnels ne militent pas pour le recours généralisé à du béton armé ni à du béton fibré. Les approches structurelles continuent donc à se faire sur le béton non armé. Des calculs théoriques de résistance des formes (MELBY \& TURK, 1997) ont été réalisés pour tenter d'apprécier et de comparer leur aptitude à résister. Toutefois, ces calculs statiques n'apparaissent pas forcément pertinents dans la mesure où le sujet porte surtout le comportement du matériau vis-à-vis des chocs et aussi de sa fatigue sous chocs répétés, l'oscillation des blocs constituant la première forme de dommage rencontré sous houle croissante. Certes, le comportement vis-à-vis des chocs, de certains types de blocs, notamment les Tétrapodes et les Dolos, a fait, plus particulièrement depuis le début des années 90, l'objet d'études spécifiques (BURCHARTH \& LIU, 1995) mais leur traduction concrète en termes de prescriptions techniques applicables sur les chantiers est quasiment inexistante. Le problème est d'autant plus délicat que le comportement du béton aux chocs dépend de la composition du béton et que les critères de performance du béton qui sont habituels comme par exemple, la résistance à la compression, ne sont sans doute pas pertinents. Il s'agit d'un sujet aujourd'hui très mal maîtrisé dans le milieu maritime.

\section{Conclusion}

Nous avons cherché à répertorier bon nombre de blocs artificiels utilisés en carapace de digues à talus depuis les années 50. En dépit d'une variété de produits, une classification a pu être proposée ainsi qu'une évaluation sommaire de leurs qualités respectives. D'un point de vue historique, l'évolution des tendances techniques a pu également être dégagée. Cette analyse a aussi été l'occasion de montrer les recoupements qu'il est possible de faire entre les formules les plus connues et de retrouver d'un point de vue théorique la composition des termes de la formule d'Hudson. Nous avons cru utile de souligner un certain nombre de difficultés ou d'incertitudes concernant les outils ou connaissances à disposition des praticiens. D'un point de vue général, il ressort de notre analyse que l'utilisation des blocs performants qui permettent de réaliser des économies substantielles nécessite a priori un soin tout 
particulier depuis les études jusqu'à la mise en œuvre car les approximations, omissions ou négligences devraient se traduire par des conséquences plus significatives sur l'état de l'ouvrage.

\section{Références bibliographiques}

BURCHARTH H.F., HUGHES S.A. (2002). Coastal Engineering Manual, Part VI Chapter VI-5-2 Fundamentals of Design. U.S. Army Corps of Engineers.

BURCHARTH H.F., LIU Z. (1995). The application of load-cell technique in the study of armor unit responses to impact loads. Proceedings of the Final Workshop, Rubble Mound Breakwater Failure Modes, Sorrento, Italy.

HATTORI M., YAUCHI E., KASAHARA Y. (1999). Hydraulic stability of armor units in single cover layer. In Proc. of the international conference Coastal Structures '99, Santander, Spain, 7-10 June, 1999, Edited by LOSADA I.J., Balkema, Rotterdam.

HUDSON R. (1958). Design of quarry-stone layers for rubble mound breakwaters. Waterways Experiment Station Report, Vicksburg, USA, n 2-2.

HUDSON R. (1959). Laboratory investigation of rubble mound breakwaters. Journal of Waterways and Harbor Division 85, pp 93-119.

IRIBARREN CAVANILLES R. (1938). Una formula para el calculo de los diques de escollera. Revista de Obras Publicas, Madrid.

IRIBARREN CAVANILLES R. (1965). Formule pour le calcul des digues en enrochements naturels ou éléments artificiels. XXI Internat, Navig. Congress, Sect. 111, Stockholm

MELBY J.A., KOBAYASHI N. (1996). Incipient motion of breakwater armor units. Proc. of the $25^{\text {th }}$ International Conference on Coastal Engineering, Chap 140.

MELBY J.A., TURK G.F., (1997). Core-Loc concrete armor units. Technical report CHL-97-4. U.S. Army Corps of Engineers.

TORUM A. (1994). Wave-induced forces on armour unit on berm breakwaters. Journal of Waterways, Ports, Coastal and Ocean Engineering, ASCE, Vol 120, N’3 pp 251-268. doi:10.1061/(ASCE)0733-950X(1994)120:3(251)

VAN DER MEER J.W. (1988a). Rock slops and gravel beaches under wave attack. Thèse, Université de Delft. Aussi, Delft Hydraulics Publication n ${ }^{\circ} 396$.

VAN DER MEER J.W. (1988b). Stability of cubes, tetrapods and accropods. Design of Breakwaters, Thomas Telford. Proc. Breakwater 88 conference, Eastbourne.

VAN GENT M.R.A. (1996). Numerical modelling of the wave interaction with dynamically stable strucutures. Proc. of the $25^{\text {th }}$ International Conference on Coastal Engineering, Chap 150. 


\title{
History of concrete blocks used in breakwaters
}

\author{
Iman SAFARI ${ }^{1}$, François ROPERT ${ }^{2}$,
}

Dominique MOUAZÉ ${ }^{1}$, Alexander EZERSKY ${ }^{1}$

1. Université de Caen, UMR 6143 CNRS -M2C,

2-4 rue des Tilleuls, 140000 Caen, France

safari.iman@gmail.com

2. Société Soframarp,

4, Sq Jean Mermoz 60200 Compiègne, France.

Translated version not provided by the authors

Version traduite non fournie par les auteurs 
4.16 : Revue Paralia - Vol. 6 (2013) 